\title{
MPBUS: MODULAR POWER BUS FOR SPACE VEHICLES
}

\author{
Ricardo Martin ${ }^{(1)}$, Eduardo Remírez ${ }^{(2)}$, Javier Alonso ${ }^{(3)}$, Eran Priel ${ }^{(4)}$, Alain Carapelle ${ }^{(5)}$, Daniel Actis ${ }^{(6)}$ \\ (1) Project Manager, JMP Ingenieros, 26371 Sotés - La Rioja (Spain), ricardo.martin@jmpingenieros.es \\ (2) CEO, JMP Ingenieros, 26371 Sotés - La Rioja (Spain), Eduardo.remirez@jmpingenieros.es \\ (3) CTO, Nájera Aerospace, 26371 Sotés - La Rioja (Spain), javier.alonso@,nasp.es \\ (4) HW\&SW Engineer, Yamar Electronics, 6249220 Tel Aviv (Isarel), eran@yamar.com \\ (5) Radiation expert, Centre Spatial de Liège, 4031 Angleur (Belgium), A.Carapelle@ulg.ac.be \\ (6) HW\&SW Engineer, Centre Spatial Liége, 4031 Angleur (Belgium), d.actis@ulg.ac.be
}

\begin{abstract}
Cost reduction in the access to space is leading the space race in the launchers market. Launcher's companies are looking for a more efficient and economical way to place satellites in orbit.

MPBUS project proposes a decentralized power bus distribution with jettisonable smart batteries combined with the use of PLC technology, data over DC, achieving overall weight reduction and harness simplification, both goals in line with Next Generation Launchers (NGL) targets.
\end{abstract}

Two different bus interfaces have been developed, Battery Front End (BFE) in charge to connect power sources equipment to the power bus, and Device Front End (DFE) in charge to connect and protect power demanding equipment to the power bus. Same power wire is used for transmitting data for power management and telemetry, reducing overall data harness.

Panel demonstrator prototype integrates these front ends to functionally test the application of MPBUS concepts to the combination of both buses, unregulated $28 \mathrm{~V}$ Power Bus and Telemetry Subsystem, from VEGA launcher.

Environmental tests have been performed to MPBUS devices successfully.

\section{ABBREVIATIONS AND ACRONYMS}

$\begin{array}{ll}\text { ADC } & \text { Analogue Digital Converter } \\ \text { AFE } & \text { Analog Front End } \\ \text { AIT } & \text { Assembly \& Integration \& Testing } \\ \text { AVUM } & \text { Attitude and Vernier Upper Module } \\ \text { BER } & \text { Bit Error Rate } \\ \text { BFE } & \text { Battery Front End } \\ \text { CE-I } & \text { Conducted Emission Current } \\ & \text { Transient } \\ \text { DC } & \text { Direct Current } \\ \text { DFE } & \text { Device Front End }\end{array}$

$\begin{array}{ll}\text { DPA } & \text { Decentralized Power Bus Architecture } \\ \text { ECC } & \text { Error Correction Codes } \\ \text { EGSE } & \text { Electrical Ground Support Equipment } \\ \text { EMC } & \text { Electro Magnetic Compability } \\ \text { FPGA } & \text { Field Programmable Gate Array } \\ \text { LCL } & \text { Latch Current Limiter } \\ \text { LDO } & \text { Low Drop Out } \\ \text { MFU } & \text { Multi Function Unit } \\ \text { NGL } & \text { Next Generation Launcher } \\ \text { OBC } & \text { On Board Computer } \\ \text { PLC } & \text { Power Line Communication } \\ \text { SPI } & \text { Serial Peripheral Interface } \\ \text { TID } & \text { Total Ionizing Dose } \\ \text { TRL } & \text { Technology Readiness Level } \\ \text { VEGA } & \text { Vettore Europeo di Generazione } \\ & \text { Avanzata }\end{array}$

\section{INTRODUCTION}

While the PLC technology is widely used and accepted in the industrial sector, its application for space is still in development stages, [1], [2]. However the interest for this technology and their application for launchers is raising due to adequate matching to several NGL requirements, such as mass reduction, harness optimisation and AIT cost reduction [3].

The MPBUS project has developed a new decentralized power bus architecture (DPA) applied to launchers, introducing the Smart Battery concept, combining distributed batteries with Battery Front Ends (BFE) connected to the power bus along the launcher, allowing the use of multi-power sources along the power bus and to maintain power management network permanently monitored using PLC over same DC power wires as communication path.

Smart Batteries can be jettisoned following the launcher's stages separation schedule and MPBUS can adapt to the new power bus layout after every stage separation. 
MPBUS can also manage, with DFEs, the protection and connection/disconnection of the different loads to/from de power bus.

The MPBUS PLC maximum rate is $3 \mathrm{Mbps}$, the power network management consumes only $5 \mathrm{Kbps}$, so there are almost $3 \mathrm{Mbps}$ to be used as data payload coming from other spacecraft subsystem. In MPBUS prototype, Telemetry subsystem data, non-critical subsystem sited along all the launcher's length, has being used as payload data in PLC. Every Telemetry node is connected to the MPBUS through a DFE to be both powered and data connected using the power wires.

MPBUS combination of DPA and PLC applied to launcher typology achieve several advantages vs traditional power distribution (point to point), as power harness reduction and elimination of data wires for payload subsystem (Telemetry) data transmission.

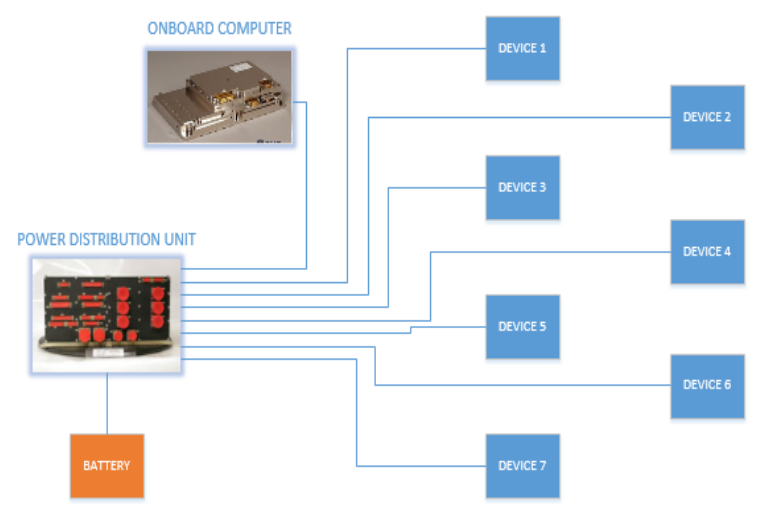

Figure 1. Traditional point to point power distribution

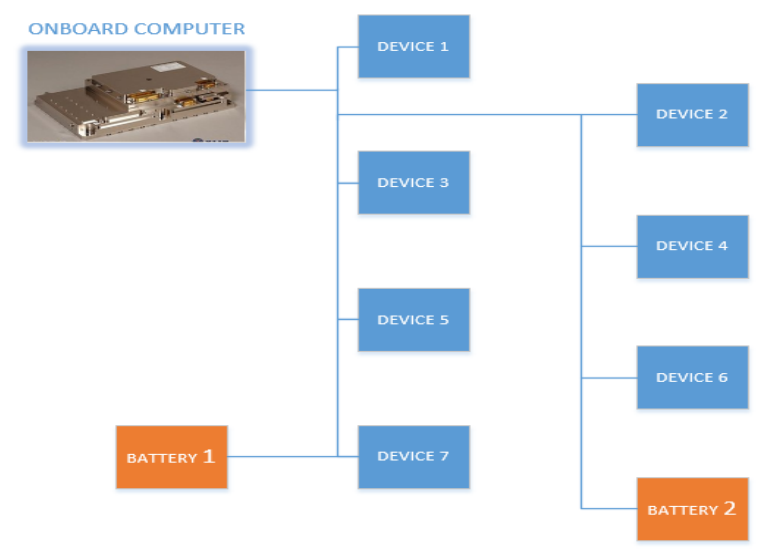

Figure 2. MPBUS decentralized power distribution

These features result in weight, volume and complexity harness reduction and thus in a greater harness design flexibility and an AIT cost reduction.

\section{DESIGN}

The MPBUS is based in a bus topology with one master node and slave nodes. Communication is based on a Token Pass technique managed by the master node with limited access time to the bus for every slave node, accessing to the bus sequentially according to a unique address. Every message in the bus is broadcast to the rest of nodes and each node process it accordingly.

The current implementation of the MPBUS in the final prototype allows one master and up to 16 slaves, but there is no theoretical limitation for maximum amount of nodes into the bus. The protocol is composed of two different types of frames: master and slave. The master frame is a broadcast message received for all nodes with a command for a single node, telemetry configuration for all nodes and telemetry data from the master node. The slave frame is a broadcast message with information to maintain the token pass, answer to master commands and telemetry data exchange. Figure 3 shows a typical MPBUS communication oscilloscope screenshot, where nodes response can be observed as a carrier frequency over $28 \mathrm{Vdc}$.

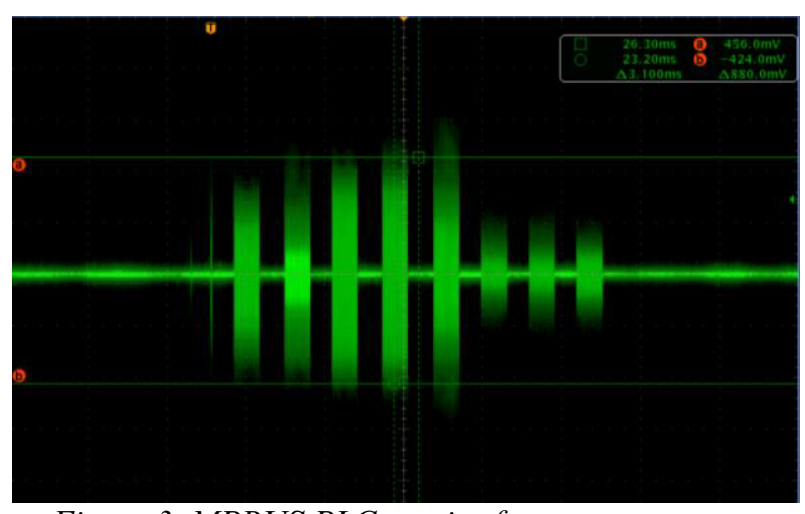

Figure 3. MPBUS PLC carrier frequency response

Distributed power is achieved by connecting /disconnecting the batteries to the two wires power bus through the BFE interfaces. Make-before-break technique is used for switching on/off batteries to the bus, two batteries can be connected to the bus at the same time due to the use of a protective diode in each $\mathrm{BFE}$, ensuring that there is always a battery supplying energy to the bus, eliminating voltage gaps in power source transitions.

Different loads or energy demanding devices can be powered/unpowered when connected/disconnected to the bus through DFE interfaces. Each DFE has its own protections, based in Latch Current Limiter (LCL) circuit, following [4] specification. 


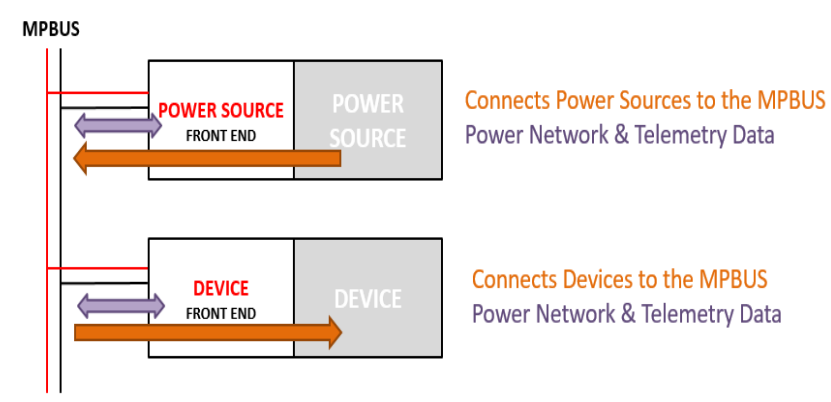

Figure 4. BFE \& DFE MPBUS interfaces

For the MPBUS final prototype, both BFEs and DFEs were designed according to VEGA launcher specifications [5].

\subsection{Battery Front End}

The Battery Front End is the interface that allows the connection/disconnection of a battery to/from MPBUS. The BFE electronics is always powered from its own battery and it is composed of the following main modules: Power Source Management, Power Supply, Control Logic, Communications and Acquisition.

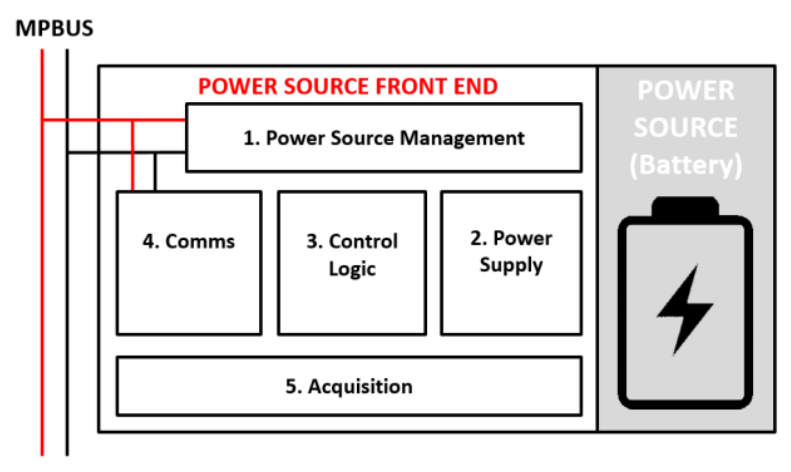

Figure 5. BFE interface

The Power Source Management is the circuit that connects/disconnects the battery to/from MPBUS. It is composed by: switching circuit, on/off sensor and current sensor.

The Power Supply is connected directly to the battery and provides the different voltage levels needed by the front end components: $3,3 \mathrm{Vdc}$ and $1,5 \mathrm{Vdc}$. It is composed by a protection circuit, DC/DC converters and LDOs regulators.

The Control Logic subsystem, based in a Microsemi FPGA ProAsic3 A3P600, is the brain of the interface and manages the different functions of the module (conection/disconection of the battery, communications and acquisition).

The Communications subsystem, the DCB3M modem, is the PLC core and it has been developed using a Microsemi FPGA SmartFusion2 M2S050. It is connected to the MPBUS and powered from the Power Supply module.

The DCB3M modem is based in a phase modulated (QPSK) narrow band carrier that protects the data with several levels of error correction codes. In addition, unique signalling technology allows arbitration over noisy and severely attenuating channels.

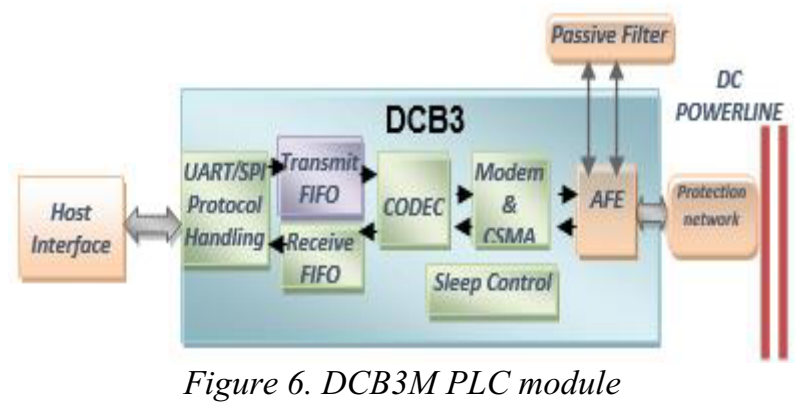

The modem implementation consists of analogue front end (AFE) that gets the analogue signal from the powerline, filter the carrier signal from the powerline noise and convert it into a digital bit stream. The digital part implemented in the FPGA recovers the digital stream into the phase-modulated carrier and decode the data from the noise. Three levels of Error Correction Codes (ECC) provide the user the ability to select the most effective ECC that mitigates the noise on the powerline.

The Communications module has a selectable carrier frequency channel $(13 \mathrm{MHz}$ to $30 \mathrm{MHz}$ in steps of $100 \mathrm{kHz}$ ), a selectable ECC level, resulting in an effective transmission rate from $0.9 \mathrm{Mbps}$ up to $3 \mathrm{Mbps}$. This module is managed through a SPI channel by the Control Logic and provides the ability to select the operating carrier frequency. A software tool has been developed to sweep the entire frequency range and map the attenuation between the nodes of the final prototype as function of the carrier frequency in order to find the channels with the lowest signal attenuation.

A study about the integration of the Communication module into an ASIC has been performed in order to assess its feasibility.

The Acquisition system acquires relevant data from the power network (battery voltage, bus voltage, current, battery cells temperature...), subsystems and external sensors. All this data is managed by the Control Logic and sent through the MPBUS by the Communication module.

For the MPBUS final prototype, the BFE has being designed to work under three different operating modes: 


\begin{tabular}{|c|c|c|}
\hline Operating Mode & Battery & Telemetry Data \\
\hline IDLE & Disconnected & No \\
\hline TLM & Disconnected & Yes \\
\hline BATTERY ON & Connected & Yes \\
\hline
\end{tabular}

Figure 7. BFE operating modes

\subsection{Device Front End}

The Device Front End (DFE) is the interface that protects the MPBUS from load device anomalies and vice versa. DFE allows the control of the connection/disconnection of a load to/from MPBUS. The electronics of this front end is always powered from the MPBUS and it is composed of the following main modules: Load Management, Power Supply, Control Logic, Communications and Acquisition.

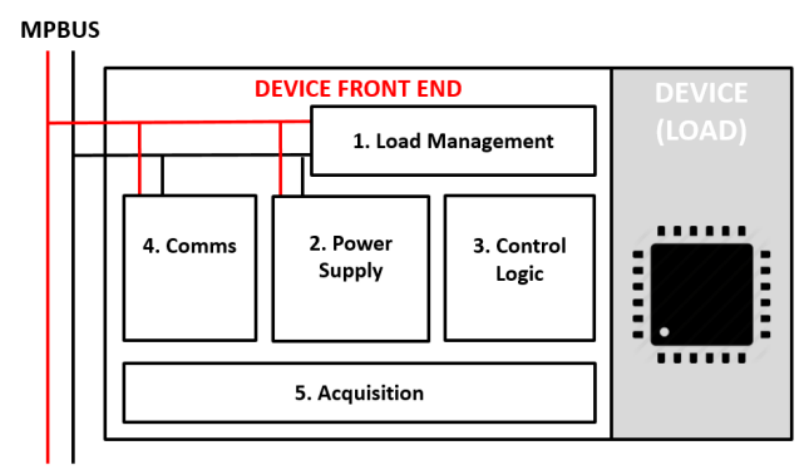

Figure 8. DFE

The Load Management is the circuit that connects/disconnects the device to/from MPBUS and it has a protection built-in LCL. It is composed by: protection circuit, switching circuit, on/off sensor and current sensor.

The Power Supply is connected directly to the MPBUS and provides the different voltage levels needed by the front end: $+5 \mathrm{~V},-5 \mathrm{~V}, 3,3 \mathrm{~V}$, and $1,5 \mathrm{~V}$. It is composed by a protection circuit, DC/DC converter and LDOs.

The hardware of the Control Logic is similar to the one in the BFE but with different firmware. Rest of DFE are equal than those than BFE.

For the MPBUS final prototype, the DFE has being designed to work under the three different operating modes as for the BFE.

\section{MPBUS PROTOTYPE}

To assess the MPBUS, two different prototypes have been manufactured. First proto was in charge to demonstrate basic front ends functionalities and served as basis for the final proto.
For final proto MPBUS assessment, unregulated 28V power bus and Telemetry subsystem from VEGA launcher were selected to be implemented according to new MPBUS concepts. This new architecture replicates them in a simplified manner on a 6 meters long panel demonstrator, simulating VEGA launcher different stages and interstages.

Wire length placed at stages has same length than real VEGA stages (total 35 meters). The nodes placed at interstages simulate real VEGA avionics load devices (OBC, UCTM, UCATs, IRS TVCs), demanding from MPBUS power sources up to $23 \mathrm{~A}$.

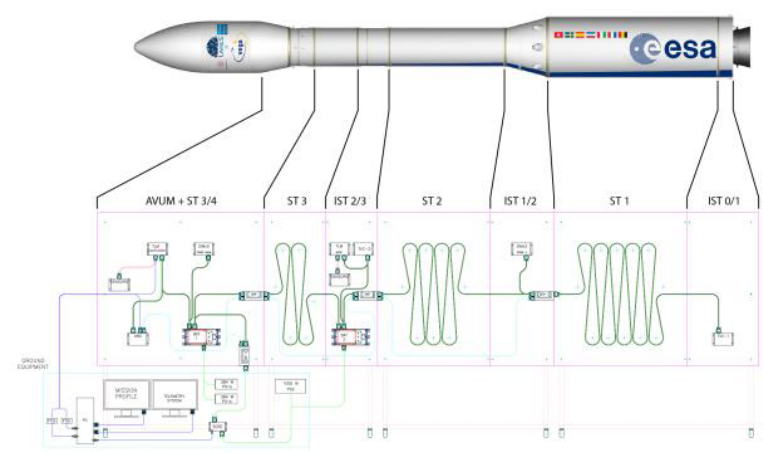

Figure 9. MPBUS Final Prototype layout

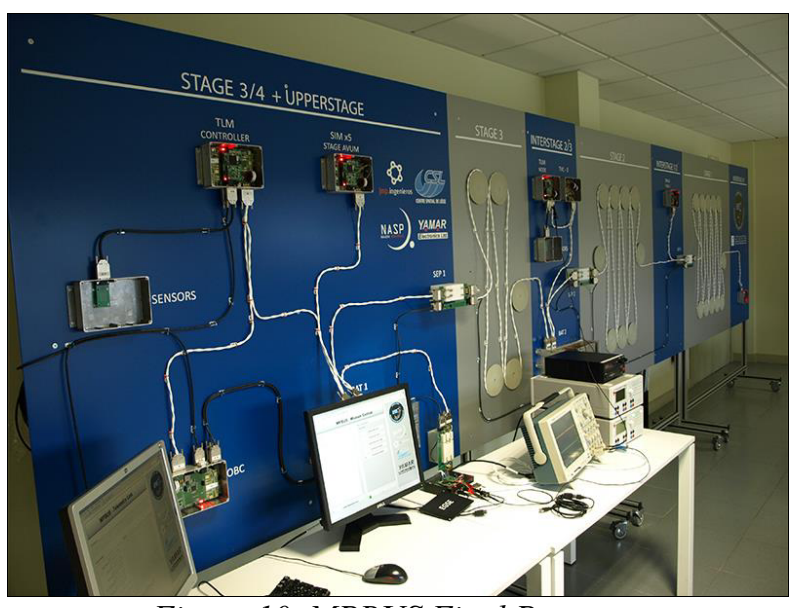

Figure 10. MPBUS Final Prototype

Stages are connected by 4 controlled bus separators based on electromechanical spring-loaded solenoid that can simulate stages jettison, allowing MPBUS to be splitted into several sections while launcher's mission is performed. 


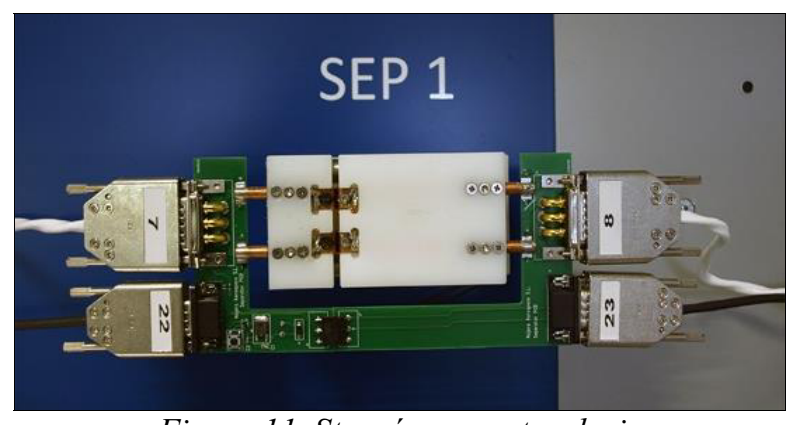

Figure 11. Stage's separator device

EGSE subsystem, external to the panel, simulates the VEGA connection to ground equipment allowing to command and control the MPBUS from ground prior to lift off phase. The EGSE part is composed by one special MPBUS node developed as a support equipment that works as a gateway between the PC used as a control unit in the EGSE and the MPBUS panel demonstrator.

There are 3 different power supplies $(28 \mathrm{~V})$ in the final prototype, one external power supply at the EGSE simulating launcher powering when at ground phase, BAT2 node at interstage $2 / 3$ powered by external power supply and BAT1 node at upperstage powered from Liion battery.

There are 7 different nodes simulating VEGA avionics devices, each one is capable to simulate load energy consumption for up to five VEGA devices and it can handle the switching of every of these loads separately.

BAT1 and BAT2 BFE and TVC3 DFE have been designed and manufactured using components with space equivalent. Rest of nodes have been designed and implemented based on industrial components for cost and time constraints.

The MPBUS harness is a two wire shielded twister PTFE insulated AWG 12 cable, selected following [6] and DB15 3W3 connectors with central pin not connected. Auxiliary industrial DB9 connectors and cables, out of the MPBUS core scope, are used as ground-launcher link wires for simulating separator's signal and wired telemetry link between UCTM and EGSE.

\subsection{Battery nodes}

Real VEGA launcher has two batteries [7], 55V and $28 \mathrm{~V}$, placed at the upperstage (AVUM). The $28 \mathrm{~V}$ battery is made of Li-ion SAFT MP176065 cells in a $8 \mathrm{~S} 8 \mathrm{P}$ configuration. This battery powers the unregulated $28 \mathrm{~V}$ bus, which powers several equipment as OBC, UCTM, UCATs, since the EGSE umbilical disconnection at launch pad to the end of mission. When AVUM starts its programmed payload orbit injection maneuver, the $28 \mathrm{~V}$ battery capacity is drained about $25 \%$. MPBUS proposes to split the original $28 \mathrm{~V} 8 \mathrm{~S} 8 \mathrm{P}$ battery into two, one with a $28 \mathrm{~V}$ 8S2P (BAT2) configuration placed at intersatge $2 / 3$ in charge to power the bus from the EGSE umbilical disconnection to just before stage 3 separation and another battery $28 \mathrm{~V} 8 \mathrm{~S} 6 \mathrm{P}$ (BAT1) in charge to power the AVUM after stage 3 separation. This battery distribution reduces the inert AVUM mass allowing to increase the thrust-to-weight ratio for this last stage.

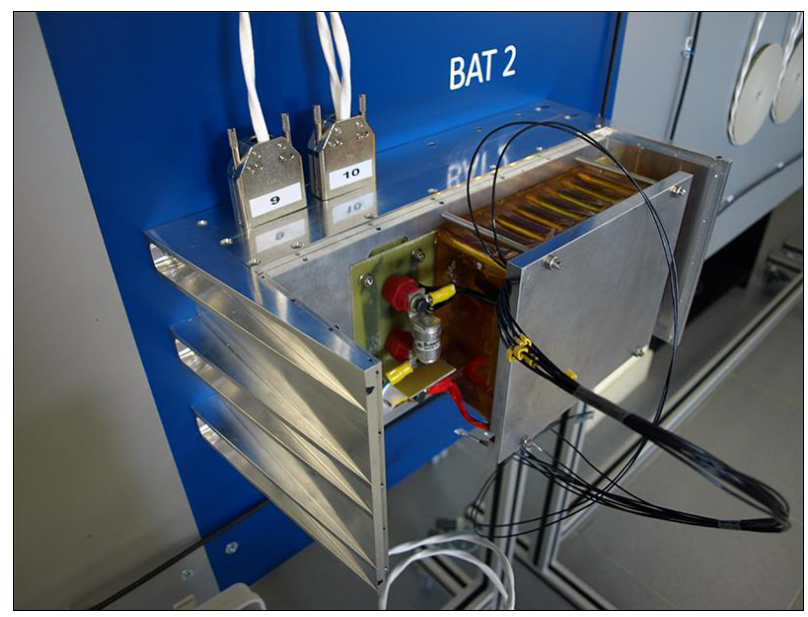

Figure 12. BAT2 node, upper enclosure removed.

Battery nodes have been designed in a two floor layout aluminum enclosure, upper floor for BAT2 accommodates $28 \mathrm{~V} 8 \mathrm{~S} 2 \mathrm{P}$ Li-ion battery and BFE board is placed at the bottom floor.

BAT2 has a NTC sensor for temperature monitoring, this analog signal is wired to the BFE where the Acquisition module sample and convert it and the Control module send it to the simulated OBC through MPBUS by the Communication module.

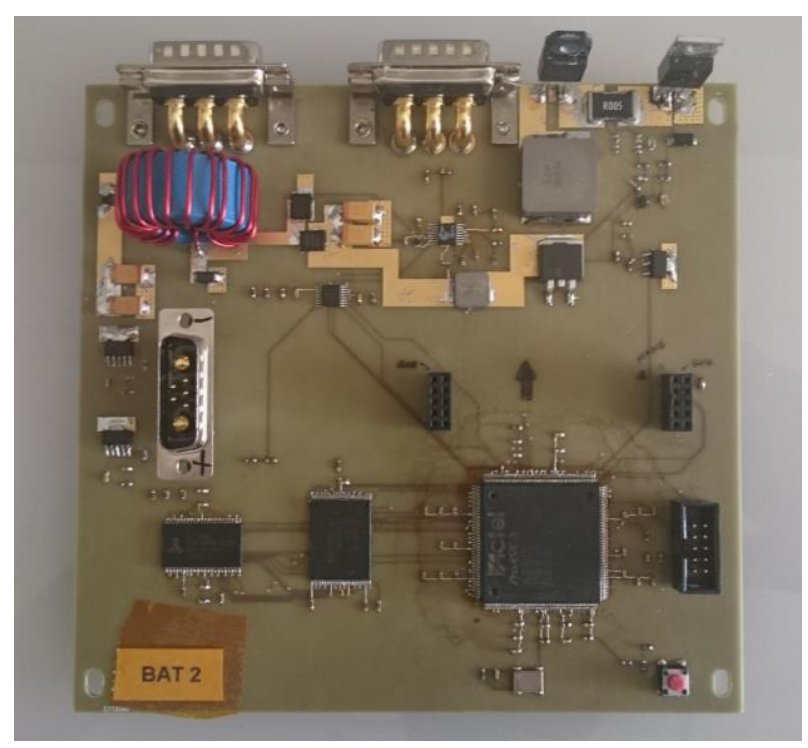

Figure 13. BAT2 BFE with PLC modem removed 
Mechanized openings in the aluminum enclosure are available for connectors to distribute power to the several MPBUS branches.

\subsection{Device nodes}

In a similar manner as with battery nodes, a front end for devices that demands energy from the MPBUS has been developed.

For MPBUS prototype 7 simulated avionics devices have been implemented onto the panel demonstrator: OBC, TLM Controller, TLM, SIMx5, SIMx2, TVC3 and TVC1. The different DFEs have loads to simulate the real consumption of the devices in the VEGA launcher. The consumptions simulated for the TVCs are the one related to the electronic control, not to the TVCs electrical actuators. The SIMx5 and SIMx2 simulated the loads of several devices of VEGA.

The system is managed by the EGSE node at the beginning of the mission simulation. Once the EGSE is removed, the OBC becomes the master of the MPBUS.

The TLM Controller is the node that collects all data sent in the MPBUS and resend them to the EGSE station for post analysis.

Rest of devices just simulates the energy consumption and send their telemetry data.

TVC3 DFE has been designed and manufactured using components with space equivalent. A LCL class 3 circuit has been implemented. Rest of DFEs are developed with industrial components.

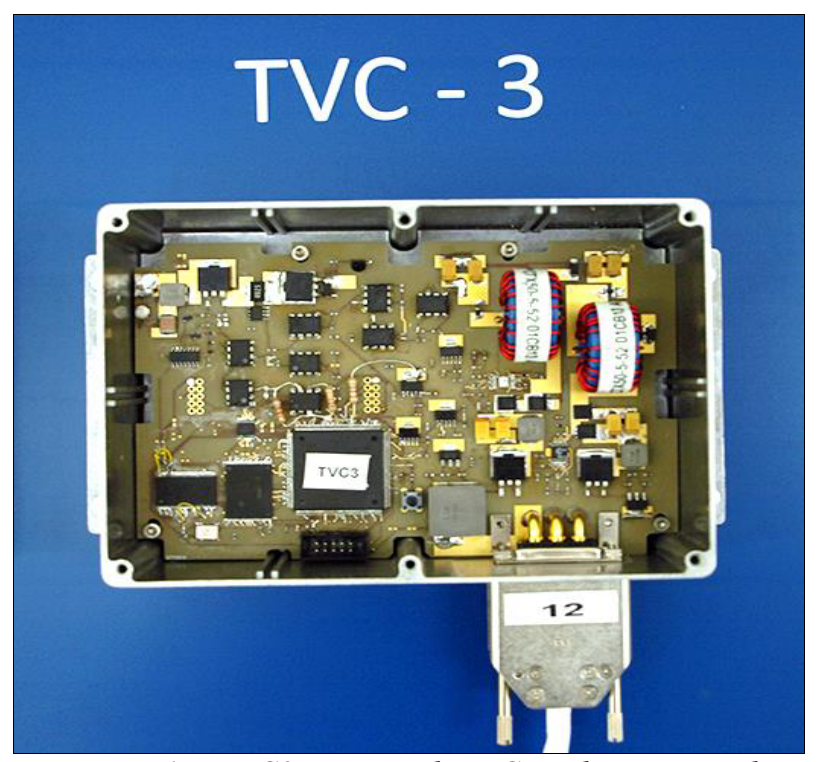

Figure 14. TVC3 DFE with PLC modem removed

\section{TESTS RESULTS}

The BFE and DFE have been functionally tested into the panel demonstrator at NASP facilities and environmental tests over single nodes have been performed at CSL facilities to assess their compatibilities with VEGA launcher specifications.

\subsection{Functional Tests}

Three main functional tests have been performed: (i) mission test, (ii) transfer rate and (iii) word error rate. A specific testing software has been developed to manage different missions, collect and analyse data to extract test results.

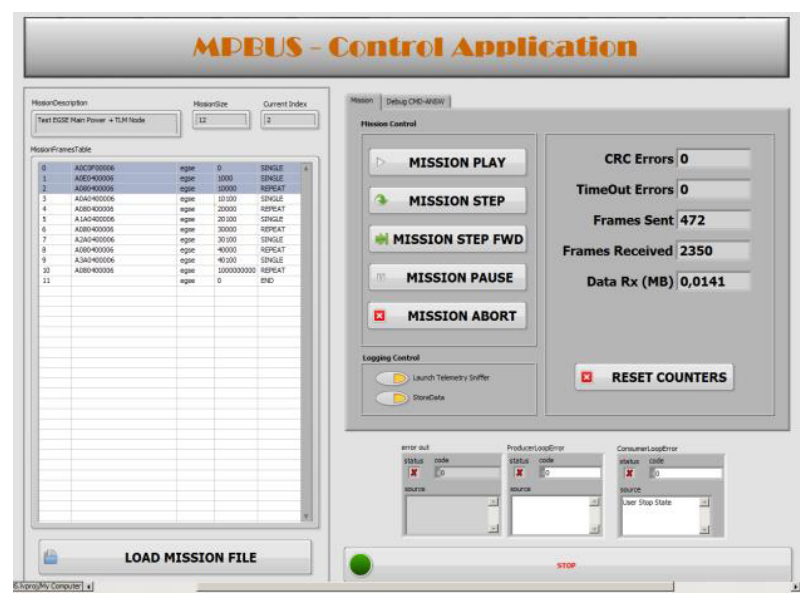

Figure 15. MPBUS test application

The MPBUS final prototype was functionally tested following a simulated typical VEGA mission, covering from previous lift off moments to end of mission phase. During the mission, device and battery nodes were communicating through the MPBUS and executed their respective connection/disconnection in a correct sequence to perform the mission. Launcher stages were sequentially jettisoned and the nodes were connected and disconnected until the last stage remained powered from its own BFE. MPBUS communications, operation modes of every node and different power distribution scenarios, up to $23 \mathrm{~A}$, were achieved successfully.

Transfer rate tests was performed with all MPBUS nodes connected configured with Telemetry mode on, maximum sample rate and hard ECC. Average transfer rate obtained was $0,90 \mathrm{Mbps}$, almost matching with real VEGA Telemetry maximum rate $1 \mathrm{Mbps}$ [8].

Word error rate tests has been performed over all possible MPBUS layout configurations after every stage separation. TLM Controller node role was to sniffer all MPBUS communications frames and send them to EGSE equipment for data post processing. Two 
different errors, CRC and timeout, have been recorded. MPBUS fails VEGA word rate restrictive specifications [9]. The MPBUS measured byte error rate is $2.35 \mathrm{E}-7$, showing MPBUS potential feasibility at this early development stage.

\subsection{Environmental Tests}

These tests were performed at CSL facilities to check BFE and DFE behaviour in VEGA/Ariane space environment in terms of vacuum, temperature, radiation and EMC.

\subsubsection{Radiation test}

The most critical radiation environment of a launcher is a flux of proton and neutron during 30 minutes of mission [10]. It has been established that in nominal solar conditions, the heavy ion aggression does not affect the success of a mission [11].

Electronic components from front ends selected for protons and neutrons testing were both FPGA, the ProAsic3 A3P600 and the SmartFusion2 M2S050 from Microsemi. 16 specimens each model has been tested.

There are mainly two types of effects induced by the radiation in electronic components: Total Ionizing Dose (TID) Effects and Single Event Effects (SEE). As SEE have to be managed at design level, tests focused on TID effects. Neutron dose can be converted to total ionizing dose equivalent value [12].

TID influence can be assessed by measuring the change in gate propagation delay [13]. FPGA design involving large number of serial inverter gates was developed and propagation delay was measured on test specimens before and after radiation.

\subsubsection{Protons}

The estimated fluence at $50 \mathrm{MeV} \mathrm{p}+$ for Ariane5 is around $1 . \mathrm{E}^{7} \mathrm{p}+/ \mathrm{cm}^{2}$ [14] for a 30 minutes mission. Four FPGA specimens for both models were tested with this fluence and energy (30 minutes irradiation). There were another specimen batch with four specimens each model tested with 3 times the fluence (three times the flux with same 30 minutes irradiation time).

Results manifest that there is no significant change in the propagation delay.

\subsubsection{Neutrons}

The test was done at a flux of $1 \mathrm{n} / \mathrm{cm}^{2 *}$ s (corresponding to the mean atmospheric environment [14]) and 3 $\mathrm{n} / \mathrm{cm}^{2 *} \mathrm{~s}$. The irradiation time was $30 \mathrm{~min}$ as the typical Ariane5 launcher mission. The selected source (Am-Be:
ISO 8529-2 Reference neutron radiations) has a (theoretical) spectrum between 1-10 MeV with a peak around $5 \mathrm{MeV}$. The flux of $1 \mathrm{n} / \mathrm{cm}^{2 *} \mathrm{~s}$ is obtained by adapting the distance between the source and the target, thanks to the $1 / \mathrm{r}^{2}$ effect. The total fluence after 30 minutes is then $1800 \mathrm{n} / \mathrm{cm}^{2}$.

As in protons test, four FPGA specimens from both models were tested with nominal dose and another four specimens from both models were tested with 3 times the dose during 30 minutes.

Results manifest that there is no significant change in the propagation delay.

\subsubsection{Thermal Vacuum test}

The MPBUS BFE has been tested into a vacuum chamber. Four thermal cycles between $-10^{\circ} \mathrm{C}$ and $+60^{\circ} \mathrm{C}$ at a pressure of less than $10^{-5}$ mbar were performed while $\mathrm{BFE}$ performed power connection/disconnection procedures and communication with another MPBUS nodes placing outside vacuum chamber.

No error occurs in the range of the test.

\subsubsection{EMC test}

BFE and DFE have been tested at the University of Liége Laboratory in a semi-anechoic chamber for EMC, following [15] and [16] procedures, to test the devices in radiative/conductive emission and susceptibility.

Except the transient current that exceeded the limit at the CE-I test, the MPBUS passed all tests.

\section{CONCLUSION}

The MPBUS solution has demonstrated the benefits of combining decentralized power distribution and PLC, achieving mass reduction, harness optimisation, complexity, risk and AIT cost reduction.

Communication over power wires has been stablished by MPBUS nodes despite to the complex environment prototype: stages separation, connectors and separator's interfaces, bus branches and wire length.

Most of the functional and environmental tests based on VEGA's specifications were successful, even at current MPBUS TRL 4, showing great potential for next development phases.

Lack of specific standards for spacecrafts using PLC technology has been detected. MPBUS developments can help to settle future standardization about this topic. 


\section{ACKNOWLEDGMENTS}

The research leading to these results has received funding from the European Community's Seventh Framework Programme (FP7/2007-2013) under grant agreement $\mathrm{n}^{\mathrm{0}} 606818$ for the MPBUS project.

\section{REFERENCES}

1. Powerlink: Two-Wire Power and Data Link. Statement of Work - Appendix 1 to ESTEC/ITT $\mathrm{AO} / 1-6774 / 11 / \mathrm{NL} / \mathrm{AT}$, Issue 1 , Rev 5 .

2. Johannes Wolf. Power Line Communications (PLC) in Space- Current Status and Outlook. Proceedings 2012 ESA Workshop on Aerospace EMC.

3. http://www.esa.int/Our_Activities/Launchers/Tech $\underline{\text { nology }}$

4. ECSS-E-ST-20-20C DIR1. Space engineering Electrical design and interface requirements for power supply. ESA-ESTEC 20 May 2015.

5. VG-SG-1-C-35-SYS Issue 5 Rev.1. VEGA Program - General Design Norms and Rules. ELV S.p.A.

\section{ECSS Detail Specification No.3901/024}

7. R. Albano, P. Brochard and F. De Coster: "Lithiumion batteries for the VEGA launcher" Proceedings of the conference on Changes In Aeronautical And Space Systems - Challenges For On Board Energy, Avignon, June 2006, France

8. VG-ST-1-C-002-SYS Issue 6 Rev. 1, VEGA Programme-Avionics System Technical Specification, page 74 .

9. VG-SG-1-C-037-SYS Issue 3 Rev.1, VEGA Program - Communication System General Specification, pp 28,30.

10. Ariane 5 User's Manual Issue 5 Rev. 1, July 2011

11. Calvet, M.C., Mazon, R., Simon, Ph., Galindo, D., Poivey, C., Garnier, P., Bourrieau, J., Cadot, B., Ecoffet, R., Acknowledgement of the Natural Radiation Environment upon the ARIANE 5 Launcher, Third European Conference on Radiation and its Effects on Components and Systems, RADECS 1995, pp. 170-174 France.

12. A.Holmes, L.Adams, Handbook of radiations effects, $2^{\text {nd }}$ edition, Oxford University Press (2007)
13. Kastensmidt F. L., Pereira Fonseca, E. C., Vaz, R. G., Gonçalez, O. L., Chipana, R. and Wirth G. I., TID in Flash-Based FPGA: Power Supply-Current Rise and Logic Function Mapping Effects in Propagation-Delay Degradation, IEEE Trans. Nucl. Sci., Vol.58, Issue 4, pp 1927-1934, 21 April 2011

14. Colas, G., Neutrons atmosphériques en avionique, Presentation to RIS, Thalès Avionics, CAL/DT/I, 20 Nov. 2002

15. VG-SG-1-C-30-SYS Is. 4 Rev.1

16. MIL-STD-461F standard 